\title{
ZNF451 Gene
}

National Cancer Institute

\section{Source}

National Cancer Institute. ZNF451 Gene. NCI Thesaurus. Code C142206.

This gene is involved in protein sumoylation. 